\title{
Mélanges de la Casa de Velázquez, 45 (1)
}

("Langues indiennes et empire dans l'Amérique du Sud coloniale ", Juan Carlos Estenssoro et César Itier [coord.]), 2015, 280 p., ill., cartes.

Isabel Yaya

\section{OpenEdition}

\section{Journals}

Édition électronique

URL : https://journals.openedition.org/jsa/14723

DOI : 10.4000/jsa. 14723

ISSN : 1957-7842

Éditeur

Société des américanistes

\section{Édition imprimée}

Date de publication : 31 octobre 2016

Pagination : 219-225

ISSN : 0037-9174

\section{Référence électronique}

Isabel Yaya, "Mélanges de la Casa de Velázquez, 45 (1) 》, Journal de la Société des américanistes [En ligne], 102-1 | 2016, mis en ligne le 05 décembre 2016, consulté le 04 septembre 2022. URL : http:// journals.openedition.org/jsa/14723; DOI : https://doi.org/10.4000/jsa. 14723

Ce document a été généré automatiquement le 4 septembre 2022.

Tous droits réservés 


\section{Mélanges de la Casa de Velázquez, $45(1)$}

("Langues indiennes et empire dans l'Amérique du Sud coloniale », Juan Carlos Estenssoro et César Itier [coord.]), 2015, 280 p., ill., cartes.

Isabel Yaya

\section{RÉFÉRENCE}

Mélanges de la Casa de Velázquez, 45 (1), (« Langues indiennes et empire dans l'Amérique du Sud coloniale », Juan Carlos Estenssoro et César Itier [coord.]), 2015, 280 p., ill., cartes.

« [Charles Quint] disait que s'il voulait parler aux Dames, il parlait Italien; que s'il voulait parler aux hommes, il parlait Français ; que s'il voulait parler à son cheval, il parlait Allemand; mais que s'il voulait parler à Dieu, il parlait Espagnol ». Dominique Bouhours, Les entretiens d'Ariste et d'Eugène, 1671.

1 Il faut avoir l'esprit roman pour suivre sans s'égarer la trame polyglotte de ce superbe dossier des Mélanges de la Casa de Velázquez où thématiques et langues de rédaction font judicieusement corps. Une introduction, six articles et un contrepoint rédigés en français, espagnol, portugais ou italien sont consacrés à la question des dynamiques linguistiques dans les espaces colonisés par les puissances ibériques à l'époque moderne. Bien que l'Amérique du Sud soit naturellement le terrain d'analyse privilégié des auteurs, les deux études finales s'inscrivent dans une perspective comparatiste plus globale. L'une porte sur le panorama linguistique dans l'océan Indien aux xvi et XVII ${ }^{e}$ siècles, tandis que l'autre aborde la prédication jésuite dans l'Italie rurale entre les $\mathrm{XVI}^{\mathrm{e}}$ et XVIII siècles. Mais n'est pas Charles Quint qui veut. Pour ma part, la lecture de ces Mélanges au titre opportun a nécessité le recours éhonté à quelques dictionnaires 
bilingues, ce qui sera perçu, je l'espère, comme un effort de communion avec les propos des coordinateurs.

2 L'introduction rédigée par Juan Carlos Estenssoro et César Itier présente les différents champs disciplinaires et les visées théoriques de cette collaboration intitulée « Langues indiennes et empire dans l'Amérique du Sud coloniale ». La philologie, l'histoire sociale des langues et l'étude des pratiques hégémoniques en situations coloniales se côtoient au fil des articles. Il s'agit pour leurs auteurs de saisir sur la longue durée les dynamiques sociales qui ont favorisé la propagation de grandes familles de langues autochtones dans l'Empire ibéro-américain au détriment de celles importées par les conquérants européens. Tant les agents coloniaux que les populations subordonnées contribuèrent ainsi à imposer le nahuatl, le quechua, l'aymara, le guarani ou le tupi comme « langues générales" en contextes pluriethniques. Dans les Andes et le Brésil, celles-ci s'imposèrent parmi les métis, mais aussi communément parmi les natifs espagnols et portugais. Pour Estenssoro et Itier, ces «langues générales» présentent des caractéristiques qui les distinguent d'autres catégories sociolinguistiques comme les langues véhiculaires, les linguae francae ou les koinès. Outils de communication comprenant plusieurs registres, elles étaient avant tout la langue d'un groupe politiquement ou démographiquement prédominant. Leur propagation à l'époque coloniale visa à imposer le monolinguisme au sein d'espaces au profil linguistique, ethnique et sociologique particulièrement hétérogène. Elles permirent ainsi la mise en place d'institutions gouvernantes communes et furent, de fait, des instruments clefs de la domination coloniale dans les Amériques. Mais par quels processus de tels phénomènes sociolinguistiques se sont-ils mis en place?

3 Afin de répondre à cette question, l'un des principaux volets exploré dans ce dossier est celui des valeurs associées aux langues amérindiennes dans la littérature coloniale, tant laïque que religieuse. Suivant cette voie, les contributeurs examinent la production textuelle qui entendit présenter les dispositions morales et civiques des populations indigènes à partir des caractéristiques attribuées à leur langue. Ces écrits opèrent une série d'oppositions entre les vertus des «langues générales» et les vices des langues dites «maternelles» ou "particulières». À l'unicité et à l'élégance des premières s'opposaient la confusion du nombre et le barbarisme des secondes. À partir de ces matériaux, Estenssoro propose d'interpréter la politique linguistique élaborée à l'intérieur de l'espace ibéro-américain comme un appareil hégémonique qui traversa l'ensemble des domaines de l'activité coloniale. En effet, dans l'Amérique hispanique, l'appartenance à l'Église était constitutive de la personne sociale. Un individu devenait un sujet plein et entier de la Couronne lorsqu'il démontrait sa Foi par la mémorisation du catéchisme. Il accédait ainsi à la justice et aux éventuelles charges publiques. Il s'acquittait en retour des obligations tributaires attachées à son statut. Or l'évangélisation se fit par le biais d'un nombre restreint de langues indigènes dont certaines acquirent progressivement le statut de «langue générale ». Au Pérou, seul le quechua reçut cette désignation en vertu de ses qualités présumées. Diffusée par les instances étatiques incas, la variante qhichwa simi bénéficiait d'une aura particulière aux yeux des colons. Langue préhispanique de l'unification politique et religieuse, propagée par une aristocratie conquérante, elle présentait les dispositions intrinsèques à la diffusion de l'œuvre coloniale. Sa propagation, comme celle des autres «langues générales ", marginalisa les idiomes régionaux. Cette homogénéisation linguistique se consolida à la suite du troisième concile de Lima (1581-1583) qui établit la normalisation 
du quechua catéchétique. Elle se stabilisa enfin au sein de l'environnement pluriethnique des reducciones et des lieux de corvée (mit'a).

4 L'émergence du nahuatl en tant que «langue générale » résulta également du travail missionnaire en Nouvelle-Espagne. Les lois de Burgos (1512) avaient initialement imposé aux ordres religieux installés dans le Nouveau Monde d'enseigner en latin ou en romance (castillan). D'après quelques témoignages coloniaux, le respect initial de cette ordonnance donna lieu à des premiers prêches à la gestuelle particulièrement expressive. Malgré les risques d'hétérodoxie inhérents à la traduction de la doctrine, les franciscains initièrent rapidement une politique d'apprentissage et de grammatisation de la « langue mexicaine » issue de la variante parlée par la noblesse de Texcoco, membre de la Triple Alliance mexica. Ce travail de lexicographie et de traduction concerna également les autres langues autochtones qui composaient la diversité du paysage linguistique mésoaméricain: mixtèque, zapotèque, tarasque, otomi, etc. Le concile de Trente (1545-1563) sanctionna ces œuvres en autorisant finalement la traduction du catéchisme en langues vernaculaires (vulgarem linguam). Parallèlement, les dispositions prises par la Couronne espagnole pour imposer l'apprentissage du castillan dans certaines écoles de Nouvelle-Espagne n'apportèrent pas les résultats escomptés. Les écrits missionnaires, franciscains au premier chef, étaient alors de plus en plus nombreux à louer l'éloquence du nahuatl et la civilité de ses locuteurs. Une hiérarchie des langues autochtones s'imposa progressivement, ce qui donna l'impulsion pour la propagation de l'enseignement de la doctrine chrétienne en nahuatl bien au-delà de son espace préhispanique de diffusion. Le 19 septembre 1580, une cédule royale reconnut le nahuatl comme « langue générale des Indiens ».

5 Soulignons qu'en toile de fond de cette hiérarchie des langues autochtones se déployaient les premières typologies de la diversité humaine qui ont marqué les débuts de l'ethnologie comparative (Pagden 1982). Ces travaux considéraient que les qualités formelles d'une langue, l'étendue de son vocabulaire et sa capacité à désigner des concepts abstraits permettaient de mesurer empiriquement la civilité de ses locuteurs. Une langue "policée ", c'est-à-dire ordonnée, régulière, à la sonorité plaisante et au lexique abondant, était à l'image de l'ordre social établi par ses locuteurs. Cette homologie formelle entre deux productions de la raison - la langue et la polis - ouvrait le champ des comparaisons. De nombreux écrits coloniaux ont ainsi assimilé les effets de la propagation du quechua et du nahuatl par les Empires inca et aztèque à l'impact civilisateur du latin dans l'Empire romain (MacCormack 2007, p. 192-201). En Occident comme en Amérique, la diffusion de ces langues aurait posé les fondations providentielles du travail apostolique.

6 Si les trajectoires coloniales du quechua et du nahuatl paraissent relativement linéaires, celles d'autres «langues générales» le sont moins. Pour exemple, Estenssoro évoque dans la dernière partie de son exposé les cas muisca et yunga. Tous deux mériteraient une étude approfondie que le format contraignant d'un article ne lui a malheureusement pas permis de présenter. Le muisca était le principal sous-groupe dialectique de la famille chibcha dont la diffusion débordait les frontières de la Nouvelle-Grenade. Au Xvi siècle, la multiplicité des variantes régionales chibcha était telle que les autorités ecclésiastiques n'y perçurent qu'une mosaïque de langues "particulières». La transmission de la doctrine chrétienne se fit donc par l'usage de ces différents idiomes. Or, d'après Estenssoro, ces pratiques missionnaires auraient bouleversé l'équilibre linguistique régional à la fin du $\mathrm{XVI}^{\mathrm{e}}$ siècle. En effet, en 1582, le muisca reçut sa première 
chaire d'enseignement à Santa Fé de Bogotá et fut reconnu "langue générale " de Nouvelle-Grenade. Ces dynamiques locales demeurent néanmoins obscures. Espérons qu'une prochaine publication abordera ce thème, ce qui permettrait également d'éclairer les processus qui ont mené à la disparition de cette langue dans le courant du XVIII siècle. Quant au yunga, bien que reconnu très tôt "langue générale", il ne connut pas d'expansion par l'évangélisation. Ce désintérêt, associé à l'absence d'une grammatisation effective, expliquerait son déclin, puis sa disparition au début du $\mathrm{xx}^{\mathrm{e}}$ siècle.

7 L'influence des pratiques missionnaires sur la trajectoire des "langues générales" apparaît donc essentielle. Notons qu'elle se vérifie dans de nombreux autres cas comme celui de l'araucan (parfois mapuche), la « langue générale du royaume du Chili » et, plus significativement, celui du siona, la «langue générale » de famille tucano diffusée dans les missions de la rivière Putumayo. Estenssoro démontre ainsi que le projet colonial ibérique s'est érigé sur des dynamiques linguistiques qui se sont progressivement transformées par l'instauration de hiérarchies ethniques. Celles-ci furent véhiculées notamment par un système discriminant de dénomination des langues autochtones.

La contribution d'Itier offre des éléments de comparaison entre les pratiques hégémoniques des puissances ibériques et celles de l'Empire inca. Il avance que le glottonyme qhichwa, qui en vint à désigner la «langue générale » du Pérou colonial, appartenait déjà à un système de classification des langues en usage sous les Incas. Ces derniers dénommèrent les langues en fonction de la sphère environnementale de leurs locuteurs. En effet, à l'arrivée des Espagnols, les Andins distinguaient déjà trois étages écologiques: les terres chaudes de la yunka, les vallées montagnardes tempérées de la qichwa et les steppes d'altitude de la sallqa (ou puna). Cette tripartition était également associée à un système de classification des humains et des produits de culture. L'analyse des écrits coloniaux montre ainsi que l'administration inca employait la désignation qhichwa simi pour décrire la " langue des terres tempérées », tandis que le terme yunga se rapportait à la langue des terres chaudes situées entre Lima et Guayaquil. Enfin, hawa simi désignait « la langue des hauts-plateaux », le mot hawa indiquant ce qui est en hauteur, à l'extérieur. Itier apporte une dimension sociologique fondamentale à ces données philologiques. En effet, une telle nomenclature maintenait une dissymétrie entre les langues véhiculaires des habitants des vallées, nommées en référence à leur écosystème, et celles des résidents des hauts-plateaux, désignées par une catégorie spatiale générique (hawa). Cette dissymétrie renvoyait à l'importance stratégique de ces groupes au sein de l'économie politique de l'empire. Parmi eux, les hawa runa désignaient originellement les populations autochtones de la région de Cuzco, implantées dans les hauts-plateaux depuis plusieurs siècles. Ils y pratiquaient des activités de subsistance au rendement pauvre : le pastoralisme et la culture en terrain sec. Or, la formation de l'État inca reposa en grande partie sur des stratégies de déplacement de populations. L'implantation des premiers migrants eut lieu dans les vallées tempérées des alentours de Cuzco. Les dirigeants incas y installèrent une main-d'œuvre d'origine variée afin d'exploiter des terres jusque-là délaissées par les populations natives. Les nouveaux arrivants y diffusèrent une variété de qhichwa simi qui devint la « langue générale » coloniale. Ainsi, le système inca de désignation des langues opposait les langues véhiculaires parlées à l'intérieur de cet espace économique riche et les langues autochtones de l'« extérieur ». Par cet argument minutieux, Itier étaye la proposition initiale d'Alfredo Torero selon laquelle l'appellation qhichwa pour désigner la «langue générale» était d'origine indigène, et non espagnole. 
9 En contrepoint de ces études sur l'aire hispanique, trois articles sont consacrés aux dynamiques linguistiques qui ont présidé à l'apparition et à la stabilisation des « langues générales » au Brésil. Guillaume Candela et Bartomeu Melià examinent la trajectoire du tupi et du guarani, deux langues de même famille, que les écrits coloniaux ont progressivement distingué au fil de quelques décennies. À partir de l'analyse croisée de témoignages locaux, ils proposent d'identifier quatre étapes dans ce processus. À partir de 1527, quelques explorateurs s'aventurèrent le long de la côte de l'actuelle Santa Catarina vers l'embouchure du río de la Plata. Leurs écrits évoquent des groupes (cario, guaraní et tupi) qui partageaient un même ethos guerrier, mais pas la même langue. Une quinzaine d'années plus tard, plusieurs témoignages affirmaient cependant que cario et guaraní étaient deux dénominations d'une même population de langue commune, désignée pour la première fois par le terme guarani. Celle-ci était alors utilisée comme langue véhiculaire entre les différentes communautés qui peuplaient le territoire de l'actuel Mato Grosso et du Pantanal. D'après Guillaume Candela et Bartomeu Melià, la diffusion du guarani serait due à l'expansion démographique de ses locuteurs par le biais de conquêtes et de migrations. À partir des années 1550, les alliances contractées entre les puissances ibériques et les populations indigènes infléchirent davantage ce paysage. Une distinction s'opéra dès lors entre les guaraní vassaux de la Couronne espagnole et les tupí sujets du roi du Portugal. Enfin, ce fut un jésuite qui, en 1591, décrivit pour la première fois le guarani comme « langue générale » du Brésil.

Une même approche diachronique traverse l'étude de Charlotte de Castelnau-L'Estoile qui examine l'apparition de la "langue générale» dans le Brésil du $\mathrm{XVI}^{\mathrm{e}}$ siècle. Elle démontre que la mise en place progressive de cette expression émergea des conflits entre colons et missionnaires pour la juridiction des populations indigènes. Installés depuis 1549 à Bahia, les premiers jésuites du Nouveau Monde se lancèrent dans l'apprentissage de la langue locale qu'ils nommèrent « langue du Brésil » ou « langue des Indiens ». Le père José de Anchieta en ébaucha rapidement la première grammaire, diffusée sous forme manuscrite à partir de 1560 au plus tard. Son travail fut cependant vivement critiqué par le grammairien Pêro de Magalhaes de Gândavo dont les écrits visaient à compromettre les caractéristiques de la langue indienne. Il argumenta notamment que cette langue manquait de lettres et qu'elle ne permettait pas de transmettre des concepts abstraits. Pour les délateurs de la cause indigène, une telle déficience linguistique était synonyme de faiblesse de raison et justifiait la mise en esclavage de ses locuteurs. Les conflits entre colons et jésuites pour la tutelle sur la main-d'œuvre locale s'amplifièrent à partir de 1570, lorsque s'intensifia le peuplement portugais du Brésil. Chaque faction usa d'arguments linguistiques pour asseoir sa position. Les missionnaires estimaient que les peuples du littoral, malgré certaines inimitiés, partageaient une même langue aux qualités propres à la traduction de la doctrine chrétienne. Ils souhaitaient la propager à l'intérieur des terres pour pacifier et évangéliser les nations insoumises. Pour les colons, cette langue était défectueuse et, de fait, entrainait la pratique du paganisme. Le projet politique missionnaire l'emporta finalement. La grammaire d'Anchieta parut en 1595 et les jésuites reçurent dès lors la responsabilité exclusive des expéditions à l'intérieur des terres. En 1606, la parution de la biographie d'Anchieta couronna ce succès et introduisit pour la première fois l'expression « langue générale » pour décrire le tupi. Castelnau-L’Estoile dégage ainsi les enjeux économiques et missionnaires qui ont participé à l'élaboration rhétorique de la « langue générale » du Brésil. 
11 Cândida Barros prolonge cet examen par l'analyse de la catégorie «tapuya » qui, dans la littérature coloniale, désigna les Indiens du Brésil qui ne possédaient pas de "langue générale ». Les écrits jésuites des XVII et XVIII ${ }^{\mathrm{e}}$ siècles associaient ce terme à la barbarie et à la bellicosité, tandis qu'ils louaient les valeurs positives de la « langue générale » qui épaula l'évangélisation, le commerce et les conquêtes. Barros atteste par ailleurs qu'une transformation sémantique s'opéra au XVIII ${ }^{\mathrm{e}}$ siècle à la suite d'une politique linguistique de «tupinisation» des Tapuya. Le terme finit ainsi par désigner les locuteurs de la « langue générale ».

Ce dossier se clôt par une ouverture comparatiste. Luís Filipe Thomaz reconstitue la diffusion progressive du portugais dans la mosaïque linguistique qu'était l'océan Indien $\mathrm{du} \mathrm{XVI}{ }^{\mathrm{e}}$ siècle. Il indique que le portugais y coexista d'abord avec le malais comme langue véhiculaire, puis le supplanta au XVII ${ }^{\mathrm{e}}$ siècle lorsqu'il servit couramment de langue de traité entre puissances européennes et formations politiques locales. Bernadette Majorana discute enfin la mise en place d'un langage figuratif de la prédication jésuite dans l'Italie rurale de l'époque moderne. Elle décrit la gestuelle oratoire et la prosodie comme des actions performatives constitutives de la transmission du message apostolique dans un espace linguistique hétérogène.

Cette belle compilation d'articles érudits éclaire les dynamiques linguistiques qui ont présidé à la diffusion de certaines langues autochtones dans l'Empire ibéro-américain. L'accent est mis sur l'influence des politiques missionnaires, sur l'impact de la grammatisation des langues et sur la trame discriminante des typologies linguistiques qui ont inauguré les débuts des sciences de l'Homme. Tout aussi récemment, l'ouvrage coédité par K. Zimmermann et B. Kellermeier-Rehbein (2015) proposait une réflexion sur les liens entre colonialisme et linguistique missionnaire à partir de cas amérindiens et africains. Ces derniers exemples, et en particulier celui du swahili, ouvrent des axes comparatistes stimulants avec les " langues générales » examinées dans ce dossier. Une telle ouverture pose également les fondations d'autres champs d'investigation. Car si l'analyse de la littérature coloniale éclaire la grande histoire de l'impulsion missionnaire dans la propagation des "langues générales », elle demeure silencieuse sur l'agentivité des populations subalternes dans ce processus. Une approche microhistorique permettrait de saisir la réception de ces langues au sein des petites sphères sociales et de dégager les intérêts locaux qui ont facilité ou entravé la diffusion de ces langues.

\section{BIBLIOGRAPHIE}

PAGDEN Anthony

1982, The Fall of Natural Man. The American Indian and the Origins of Comparative Ethnology, Cambridge University Press, Cambridge.

MACCORMACK Sabine

2007, On the Wings of Time. Rome, the Incas, Spain, and Peru, Princeton University Press, Princeton. 
ZIMMERMANN Klaus et Birte KELLERMEIER-REHBEIN (éds.)

2015, Colonialism and Missionary Linguistics, De Gruyter, Berlin/Boston.

\section{AUTEURS}

ISABEL YAYA

Laboratoire d'anthropologie sociale 